\title{
Bacteriemia por Enterococcus resistente a la vancomicina en un niño con leucemia mieloide aguda: tratamiento satisfactorio con daptomicina

\author{
Vancomycin-resistant Enterococcus bacteremia in a child with acute myeloid \\ leukemia: Successful treatment with daptomycin
}

\author{
Dra. Ayşe Büyükcam ${ }^{a}$, Dra. Eda Karadă̆ Öncel ${ }^{a}$, Dra. Yasemin Özsürekçi ${ }^{a}$, Prof. Dr. Ali B. Cengiz ${ }^{a}$, \\ Prof. Titular Dr. Barış Kuşkonmaz y Prof. Dra. Banu Sancak
}

\begin{abstract}
RESUMEN
Las infecciones enterocócicas multirresistentes pueden ser un problema serio en los pacientes pediátricos y causarles complicaciones importantes o la muerte, en particular, en los casos de enfermedades subyacentes graves concomitantes. Las opciones de tratamiento en los niños son limitadas en comparación con las de los adultos. En este artículo, presentamos el caso de un niño de 3 años con leucemia mieloide aguda (LMA)-M7 y bacteriemia por Enterococcus resistente a la vancomicina, que se trató satisfactoriamente con daptomicina. La daptomicina puede ser un tratamiento alternativo para las infecciones por Enterococcus resistente a la vancomicina (ERV) en los niños. Se necesitan más estudios para ampliar su uso.

Palabras clave: Enterococcus resistente a la vancomicina, niño, daptomicina, linezolid.
\end{abstract}

http:/ /dx.doi.org/10.5546/aap.2016.e432

Texto completo en inglés: http:/ /dx.doi.org/10.5546/aap.2016.eng.e432

\section{ANTECEDENTES}

Las infecciones por Enterococcus resistente a la vancomicina (ERV) son un problema clínico global en los hospitales y pueden provocar endocarditis infecciosa, infecciones en el torrente circulatorio relacionadas con el catéter, infecciones en las vías urinarias y bacteriemia de causa desconocida.

a. Unidad de Enfermedades Infecciosas Pediátricas, Departamento de Pediatría, Facultad de Medicina de la Universidad Hacettepe, Ankara, Turquía.

b. Unidad de Trasplante de Médula Ósea, Departamento de Pediatría, Facultad de Medicina de la Universidad Hacettepe, Ankara, Turquía.

c. Departamento de Microbiología Médica, Facultad de Medicina de la Universidad Hacettepe, Ankara, Turquía.

Correspondencia:

Dra. Ayşe Büyükcam: dr.aysebaktir@gmail.com

Financiamiento: Ninguno.

Conflicto de intereses: Ninguno que declarar.

Recibido: 16-3-2016

Aceptado: 23-5-2016
La bacteriemia por ERV, una de las causas principales de infecciones intrahospitalarias, ocasiona complicaciones importantes y la muerte, en especial, en niños con enfermedades crónicas graves subyacentes. ${ }^{1,3}$ La daptomicina es un lipopéptido cíclico antimicrobiano derivado de la especie Streptomyces roseosporus, que tiene actividad bactericida in vitro contra los patógenos grampositivos. ${ }^{2,4}$ La experiencia clínica con la daptomicina en pacientes pediátricos con ERV es limitada. Además, la dosis, la seguridad y la tolerabilidad del fármaco no se han establecido de forma definitiva en los niños. ${ }^{2}$ Informamos nuestra experiencia con el tratamiento de la bacteriemia por ERV en un niño de 3 años que presentaba leucemia mieloide aguda (LMA)-M7 y síndrome de Down.

\section{PRESENTACIÓN DE UN CASO}

Un paciente de 3 años con síndrome de Down y LMA-M7 ingresó a la sala de emergencias con fiebre en el cuarto mes de quimioterapia.

Anamnesis: cuando tenía 23 meses, lo llevaron a una consulta médica porque presentaba dolor óseo e incapacidad para caminar. Le diagnosticaron leucemia linfoblástica precursora de células B. Para la leucemia, se le administró el protocolo para quimioterapia Total XV de St. Jude. El dolor de pies del paciente se repitió a las 28 semanas de quimioterapia; la aspiración de médula ósea reveló LMA-M7. El protocolo del tratamiento se cambió por AML BFM 2004. En la anamnesis, también se destacaba la presencia de cardiopatía congénita (comunicación interauricular y comunicación interventricular).

Examen físico: el niño tenía fiebre (temperatura timpánica de $38,5^{\circ} \mathrm{C}$ ), se escuchaba un soplo sistólico $2 / 6$ y se detectó hepatoesplenomegalia.

Datos del laboratorio: hemoglobina de 11,8 g/ $\mathrm{dl}$, recuento de plaquetas de $190 \times 10^{3} / \mu \mathrm{l}$ recuento de leucocitos de $6700 / \mu 1$ (neutrófilos $68 \%$, linfocitos y linfoblastos $14 \%$, monocitos 
$18 \%$ ), con resultados normales en los análisis de enzimas hepáticas y de orina, y en las pruebas de la función renal. La velocidad de sedimentación globular era de $43 \mathrm{~mm} / \mathrm{h}$, y la proteína C-reactiva, de 27,2 mg/dl (normal: 0-0,8 mg/dl).

El diagnóstico de recidiva de la LMA se confirmó con la biopsia de médula ósea.

Día 1: se hospitalizó al paciente y se le comenzó a administrar meropenem para la fiebre neutropénica.

Día 7: se agregó un tratamiento empírico con teicoplanina y amikacina, debido a la persistencia de la fiebre.

Día 8: se agregó anfotericina B liposomal.

Día 10: en los días siguientes, con estos antibióticos, tuvo neumonía, por lo que se le comenzó a administrar trimetoprima y sulfametoxazol. La administración de los antibióticos continuaba debido al deterioro clínico y a la fiebre prolongada. Se sabía que el paciente presentaba Enterococcus faecium resistente a la vancomicina desde hacía ocho meses, como lo indicaba el resultado del coprocultivo. Se aisló ERV en los hemocultivos realizados en el ingreso; la sangre se extrajo a través de un catéter venoso central y de la vena periférica (las sensibilidades del ERV se muestran en la Tabla 1).

Día 10: se agregó linezolid $(10 \mathrm{mg} / \mathrm{kg} / \mathrm{dosis}$ cada 8 horas) y se le administró inmunoglobulina enriquecida con inmunoglobulina $\mathrm{M}$ (pentaglobulina 4,8 g/dosis) cuatro veces.

Día 12: se agregó ciprofloxacina, debido al progreso de la neumonía (Figura 1).

Día 13: en el tercer día de tratamiento con linezolid, todavía se aislaba ERV en los hemocultivos.

TABLA 1. ERV, sensibilidadess

\begin{tabular}{lccc}
\hline Antimicrobiano & $\begin{array}{c}\text { Método de prueba } \\
\text { de la sensibilidad }\end{array}$ & $\begin{array}{c}\text { CIM } \\
(\boldsymbol{\mu g} / \mathrm{ml})\end{array}$ & $\begin{array}{c}\text { Interpretación } \\
\text { de la sensibilidad }\end{array}$ \\
\hline Ampicilina & Sistema Vitek2 (V2S) & $\mathrm{R}$ \\
Imipenem & $\mathrm{V} 2 \mathrm{~S}$ & $\geq 32$ & $\mathrm{R}$ \\
Niveles altos de gentamicina & $\mathrm{V} 2 \mathrm{~S}$ & $\mathrm{SYN}-\mathrm{S}$ & $\mathrm{S}$ \\
Niveles altos de estreptomicina & $\mathrm{V} 2 \mathrm{~S}$ & $\mathrm{SYN}-\mathrm{R}$ & $\mathrm{R}$ \\
Ciprofloxacina & $\mathrm{V} 2 \mathrm{~S}$ & $\geq 8$ & $\mathrm{R}$ \\
Eritromicina & $\mathrm{V} 2 \mathrm{~S}$ & $\geq 8$ & $\mathrm{R}$ \\
Clindamicina & $\mathrm{V} 2 \mathrm{~S}$ & $\geq 8$ & $\mathrm{R}$ \\
Linezolid & $\mathrm{V} 2 \mathrm{~S}$ & 2 & $\mathrm{~S}$ \\
Teicoplanina & $\mathrm{V} 2 \mathrm{~S}$ & $\geq 32$ & $\mathrm{R}$ \\
Vancomicina & $\mathrm{V} 2 \mathrm{~S}$ & $\geq 32$ & $\mathrm{R}$ \\
Tetraciclina & $\mathrm{V} 2 \mathrm{~S}$ & $\geq 16$ & $\mathrm{R}$ \\
Tigeciclina & $\mathrm{V} 2 \mathrm{~S}$ & $\leq 12$ & $\mathrm{~S}$ \\
Trimetoprima y sulfametoxazol & $\mathrm{V} 2 \mathrm{~S}$ & $\geq 320$ & $\mathrm{R}$ \\
Daptomicina & $\mathrm{E}-\mathrm{test}$ & 2 & $\mathrm{~S}$ \\
\hline
\end{tabular}

Abreviaturas: CIM, concentración inhibitoria mínima.

*BioMerieux Francia.

FIGURA 1. Tratamiento antimicrobiano y resultados de los hemocultivos del paciente durante la administración de daptomicina

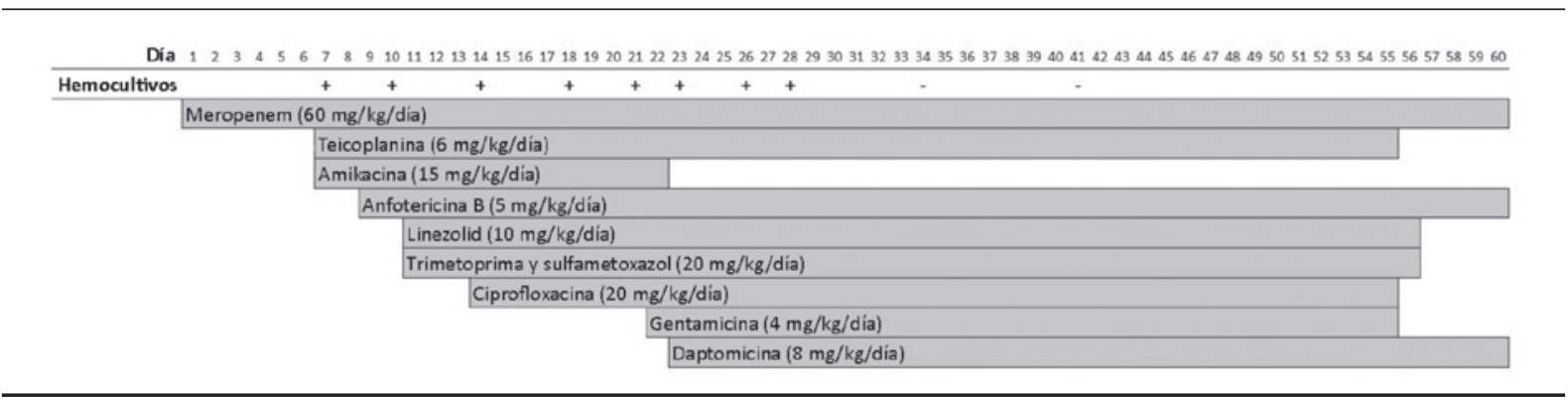


Al día 13 de tratamiento, el puerto de acceso venoso central se extrajo y se reemplazó por un nuevo catéter vascular. Ambos procedimientos se realizaron de forma simultánea, debido a la necesidad de acceso vascular. El ecocardiograma transtorácico no reveló vegetación valvular.

Día 21: la amikacina se reemplazó por gentamicina; al repetir los hemocultivos, se seguía detectando ERV.

Día 22: se agregó daptomicina $(8 \mathrm{mg} / \mathrm{kg} /$ día, dosis única) al linezolid en el día 12 de tratamiento, después del consentimiento de los padres y la aprobación del Ministerio de Salud de Turquía. La administración de linezolid continuó después del inicio del tratamiento con daptomicina.

Día 32: El hemocultivo del paciente fue negativo. El tratamiento con daptomicina continuó durante 5 semanas, período en el que los hemocultivos seguían siendo negativos (Tabla 2).

Los niveles séricos de CF estuvieron dentro de los límites normales durante el tratamiento. En los días 3, 9 y 13 después de finalizar el tratamiento con daptomicina, los hemocultivos seguían siendo negativos. Se administró el protocolo FLAG IDA previamente planificado para la recidiva de la LMA. En el día 77 de hospitalización, el paciente murió debido a infección pulmonar, hemorragia y avance de la LMA. No se detectaron microorganismos específicos durante este último episodio.

\section{DISCUSIÓN}

La bacteriemia enterocócica resistente a la vancomicina tiene un resultado desfavorable en los niños. ${ }^{5} \mathrm{Si}$ bien para las infecciones por ERV en los adultos se están utilizando diversos agentes, como quinupristina-dalfopristina, daptomicina y tigecilina, las opciones de tratamiento en los niños son limitadas, lo que representa un problema importante. Se informó que la tasa de curación clínica de las infecciones por patógenos grampositivos resistentes a los antibióticos es del $69,7 \%$ en los niños tratados con linezolid. ${ }^{6}$ En otro estudio de Garazzino y col., se logró la curación clínica o la mejoría de las infecciones por ERV con la administración de linezolid en el 66,7\% de los pacientes pediátricos. ${ }^{7}$ Sin embargo, el linezolid presenta actividad bacteriostática frente a los enterecocos, y el tratamiento puede fracasar a pesar de la sensibilidad del ERV y la extracción del puerto de acceso venoso central, como sucedió con nuestro paciente. ${ }^{8}$ En años recientes, la daptomicina ha surgido como otra alternativa para el tratamiento de las infecciones por ERV en la población pediátrica. Existen diversas publicaciones sobre la eficacia del tratamiento en niños con meningitis, bacteriemia, septicemia, endocarditis e infecciones en las vías urinarias a causa del ERV..$^{2,9,10}$ En las publicaciones, se describen diversos tratamientos combinados con daptomicina, que incluyen ampicilina,

TABLA 2. Cultivos del paciente durante el tratamiento

\begin{tabular}{|c|c|c|c|}
\hline \multicolumn{3}{|c|}{ Día de tratamiento } & \multirow[t]{2}{*}{ Otros cultivos } \\
\hline & Puerto de acceso venoso central/catéter vascular* & Vena periférica & \\
\hline 7 & ERV (+) & ERV $(+)$ & \\
\hline 8 & & & Orina (-) \\
\hline 10 & ERV $(+)$ & ERV $(+)$ & \\
\hline 14 & ERV $(+)$ & ERV $(+)$ & \\
\hline 18 & ERV (+) & ERV $(+)$ & \\
\hline 21 & $\operatorname{ERV}(+)$ & & \\
\hline 23 & & ERV $(+)$ & \\
\hline 26 & ERV (+) & ERV (+) & \\
\hline 27 & & & Líquido pleural (-) \\
\hline 28 & ERV (+) & ERV $(+)$ & \\
\hline 33 & & & Líquido pleural (-) \\
\hline 34 & - & - & \\
\hline 41 & - & & \\
\hline $63^{* *}$ & - & - & \\
\hline
\end{tabular}

* Al día 13 de tratamiento, el puerto se extrajo y se reemplazó por un nuevo catéter vascular.

** Los cultivos se realizaron después de tres días de haber interrumpido la administración de daptomicina. 
aminoglucósidos, ceftarolina, tigicilina, rifampicina y doxiciclina, que se utilizaron de forma satisfactoria para las infecciones por ERV. ${ }^{3}$ Además, ya se conoce la sinergia entre la daptomicina y la gentamicina para el E. faecium con resistencia de alto nivel a la gentamicina, ${ }^{11}$ la que ocurrió en nuestro tratamiento. Por lo tanto, no se puede generalizar el beneficio posible del uso de antibióticos múltiples.

Los datos sobre el uso de la daptomicina para pacientes pediátricos son limitados. Además, las dosis eficaces de daptomicina en los niños son diferentes a las de los adultos, y no se ha establecido con certeza la dosis óptima. En general, la daptomicina se une a una proteína en circulación y se elimina a través de los riñones. ${ }^{2}$ En algunos estudios farmacocinéticos, se ha demostrado una eliminación más rápida de la daptomicina en los niños, y parece tener una vida media más breve en los niños de 2 a 6 años, en comparación con los niños de 12 a 17 años. ${ }^{10}$ En los pacientes de menor edad, pueden requerirse dosis más altas; según las publicaciones, la dosis máxima administrada a un neonato fue de $15 \mathrm{mg} /$ $\mathrm{kg} / 12 \mathrm{~h} .{ }^{2,8}$ Nuestro paciente recibió una dosis de daptomicina de $8 \mathrm{mg} / \mathrm{kg} /$ día, la que causó la curación microbiológica y clínica de la infección, sin ningún efecto secundario.

Se han relacionado diversos factores al aumento en la incidencia de infección por ERV, que pueden atribuirse a numerosos factores que aumentan el riesgo de infección, como la colonización del tubo digestivo, el tratamiento anterior con vancomicina o fluoroquinolona, la puntuación más alta en la escala de evaluación de variables fisiológicas agudas y enfermedades crónicas APACHE II (Acute Physiology and Chronic Health Evaluation II) y el uso anterior de corticoesteroides. Además, en muchas publicaciones, se ha demostrado que la detección de ERV en la sangre, en los casos de tumores malignos hematológicos, administración de quimioterapia y bacteriemia de alto grado subyacentes está muy relacionada con la mortalidad. Sin embargo, estos factores de riesgo se demostraron en una población adulta, y los datos en pacientes pediátricos no son suficientes. ${ }^{12,13}$ Este caso de infección persistente por ERV en el torrente sanguíneo ocurrió en un niño con neutropenia grave después de la quimioterapia para la recidiva de LMA-M7, y su evolución clínica fue similar a la de los casos informados anteriormente, en especial, el desenlace desfavorable.

\section{CONCLUSIÓN}

En este informe, se destaca el abordaje terapéutico de la bacteriemia por ERV persistente en un niño con LMA-M7 y síndrome de Down, que se trató de forma satisfactoria con daptomicina. A pesar de la sensibilidad al linezolid, la fiebre se eliminó al modificar el tratamiento antimicrobiano y reemplazarlo por daptomicina, con una respuesta clínica y microbiológica favorable en este caso. Creemos que se necesitan más estudios para aclarar la farmacodinámica, la farmacocinética, la tolerabilidad y la toxicidad de la daptomicina en la población pediátrica, en especial, en los pacientes con enfermedades crónicas subyacentes.

\section{REFERENCIAS}

1. Tamma PD, Hsu AJ. Optimizing therapy for vancomycinresistant enterococcal bacteremia in children. Curr Opin Infect Dis 2014;27(6):517-27.

2. Principi N, Caironi M, Venturini F, Pani L, et al. Daptomycin in paediatrics: current knowledge and the need for future research. J Antimicrob Chemother 2015 Mar;70(3):643-8.

3. Munita JM, Murray BE, Arias CA. Daptomycin for the treatment of bacteraemia due to vancomycin-resistant enterococci. Int J Antimicrob Agents 2014;44(5):387-95.

4. Humphries RM, PollettS,Sakoulas G. A current perspective on daptomycin for the clinical microbiologist. Clin Microbiol Rev 2013;26(4):759-80.

5. Diaz Granados CA, Zimmer SM, Klein M, Jernigan JA. Comparison of mortality associated with vancomycinresistant and vancomycin-susceptible enterococcal bloodstream infections: a meta-analysis. Clin Infect Dis 2005;41(3):327-33.

6. Jantausch BA, Deville J, AdlerS, Morfin MR, et al. Linezolid for the treatment of children with bacteremia or nosocomial pneumonia caused by resistant gram-positive bacterial pathogens. Pediatr Infect Dis J 2003;22(9 Suppl):S164-71.

7. GarazzinoS, Tovo PA. Clinical experience with linezolid in infants and children. J Antimicrob Chemother 2011;66 Suppl 4:iv23-iv41.

8. Beneri CA, Nicolau DP, Seiden HS, Rubin LG. Successful treatment of a neonate with persistent vancomycin-resistant enterococcal bacteremia with a daptomycin-containing regimen. Infect Drug Resist 2008;1:9-11.

9. Ardura MI, Mejias A, Katz KS, Revell P, et al. Daptomycin therapy for invasive Gram-positive bacterial infections in children. Pediatr Infect Dis J 2007;26(12):1128-32.

10. Durand C, Brueckner A, Sampadian C, Willett KC, et al. Daptomycin use in pediatric patients. Am J Health Syst Pharm 2014;71(14):1177-82.

11. Hindler JA, Wong-Beringer A, Charlton CL, Miller SA, et al. In vitro activity of daptomycin in combination with beta-lactams, gentamicin, rifampin and tigecycline against daptomycin-nonsusceptible enterococci. Antimicrob Agents Chemother 2015;59(7):4279-88.

12. Ornstein MC, MukherjeeS,Keng M, Elson P, et al. Impact of vancomycin-resistant enterococcal bacteremia on outcome during acute myeloid leukemia induction therapy. Leuk Lymphoma 2015;56(9):2536-42.

13. Kraft S, Mackler E, Schlickman P, Welch K, et al. Outcomes of therapy: vancomycin-resistantenterococcalbacteremia in hematology and bone marrow transplant patients. Support Care Cancer 2011;19(12):1969-74. 